Research Article

\title{
Removal of Cochannel Interference Using Probabilistic Latent Component Analysis in Passive Bistatic Radar
}

\author{
Tao Ying, Xuebao Wang $\mathbb{D}$, Wei Tian, and Cheng Zhou \\ College of Electronic Engineering, Naval University of Engineering, Wuhan 430033, China \\ Correspondence should be addressed to Xuebao Wang; wangxuebao_china@163.com
}

Received 7 October 2020; Revised 3 November 2020; Accepted 21 December 2020; Published 19 January 2021

Academic Editor: Francesco Riganti-Fulginei

Copyright ( $) 2021$ Tao Ying et al. This is an open access article distributed under the Creative Commons Attribution License, which permits unrestricted use, distribution, and reproduction in any medium, provided the original work is properly cited.

\begin{abstract}
This paper examines the problem of cancellation of cochannel interference (CCI) present in the same frequency channel as the signal of interest, which may bring a reduction in the performance of target detection, in passive bistatic radar. We propose a novel approach based on probabilistic latent component analysis for CCI removal. The highlight is that removing CCI is considered as reconstruction, and extraction of Doppler-shifted and time-delayed replicas of the reference signal exploited fully as training data. The results of the simulation show that the developed method is effective.
\end{abstract}

\section{Introduction}

Recently, there has been a growing interest in passive bistatic radar (PBR) exploiting illuminators of opportunity [1-5]. Without the need for a dedicated transmitter's deployment and operation, PBR systems are significantly less expensive to implement and operate than their conventional counterparts. Due to their bistatic or multistatic configuration, PBR systems provide RCS advantages to counter stealth targets. Also, PBR systems have several advantages over conventional monostatic radars, including smaller in size, needing no additional frequency channel allocation since no dedicated transmitter is used, and immune to antiradiation missiles. Further, employing PBR systems in a multistatic configuration provides spatial diversity similar to active MIMO radar systems [6].

Many typical illuminators have already been employed for target detection in passive radars including FM radio [7, 8], Digital Audio Broadcast (DAB) [9], Digital Video Broadcast (DVB) [10-13], satellite signal [14], Universal Mobile Telecommunications System (UMTS) [15, 16], and Global System for Mobile communication (GSM) $[17,18]$. In addition, several studies have looked at the use of one potential illuminator of opportunity, rapidly growing in coverage, which is related to wireless local area networks
(LAN) [19-22]. Zhao et al. [23] employ the radio in HF band $(3-30 \mathrm{MHz})$ to achieve wide-area moving target detection and ocean remote sensing. In this paper, we will consider DVB-T transmitters as illuminators of opportunity. They have ubiquitous spatial coverage, are permanent in time, and have a thumbtack-like function due to the noise-like behavior of the orthogonal frequency division multiplexing (OFDM) modulation used. It should be noted that the developed approach in this paper can be applied to any transmission of opportunity.

The weak signal reflected from the target is received by PBR's surveillance channel with a reference channel using a directive antenna steered towards the exploited illuminator of opportunity. Usually, the target echoes may be masked by the small fraction of the direct signal received by the side lobe of the antenna of the surveillance channel, strong clutter, or multipath echoes, all of which can be considered as Doppler-shifted and time-delayed replicas of the reference signal from the transmitter of opportunity, as well as injected with CCI from other nuisance illuminating sources such as intended jamming and involuntary disturbance. Indeed, weak target echoes may be masked by the other echoes from other targets of a higher level in large rangeDoppler separations. Therefore, it is very significant to remove the disturbance for target detection, and there are 
increasing studies focusing on the solution to this problem. Kulpa and Czekała [24] present a method of adaptive removal of strong echoes from the received signal for the case when strong echoes shadow a distant, weak target echo. Raout et al. [12] achieve space-time clutter rejection and target passive detection using the amplitude and phase estimation method. An algorithm exploiting multichannel adaptive filters for sea clutter cancellation in PBR is proposed in [25]. Colone et al. [26] examine the problem of cancellation of direct signal, multipath, and clutter echoes in PBR and propose a multistage processing algorithm for disturbance removal and target detection. However, most of the above literature studies mainly focus on the cancellation of the direct signal, strong clutter or multipath echoes, and the echoes from other vital targets, without taking into account the problem of removal of CCI in a PBR due to spectrum congestion in the frequency range from VHF to $6 \mathrm{GHz}$ in the complex electromagnetic environment. CCI from other transmitters may enter the surveillance channel from the main lobe or side lobe of the receiving antenna and degrade the performance of target detection of PBR systems. To better play a role in the future war, it is equally crucial for PBR systems to preprocess signals and achieve CCI's cancellation from other nuisance transmitters. Therefore, we focus on the solution to this problem.

In recent years, the latent variable model has been a subject of research. Scholars have proposed a variety of latent variable models [27-29]. Probabilistic latent component analysis (PLCA) is discussed and applied to the separation of sounds from single-channel mixtures in [30] and radar signal detection in [31]. Likewise, the cancellation of CCI can be considered as the separation of the desired signal from the surveillance channel mixtures in a PBR. Moreover, the reference signal can be used as training data for PLCA.

Consequently, in order to make full utilization of the reference signal without increasing the complexity of the receiving system, PLCA is employed for the removal of CCI. First, in this paper, the problem of disturbance removal in the complex electromagnetic environment is proposed. Below, the PLCA method for signal preprocessing to achieve the cancellation of CCI is developed, which explicitly models the collected signal as a mixture of marginal distribution products. The estimation of the most appropriate marginal distributions, performed using the expectation-maximization algorithm, is employed selectively to reconstruct the desired signal, and eventually, the CCI suppression is achieved.

The paper is organized as follows. Section 2 describes the addressed problem. PLCA is introduced, and the PLCA method for the removal of CCI is developed in Section 3. Section 4 gives the simulation results. Finally, Section 5 provides the conclusion.

\section{The Problem}

The geometry of PBR is depicted schematically in Figure 1. The reference and surveillance antennas are assumed to be colocated with the reference antenna steered toward the transmitter and the surveillance antenna pointed in the direction to be surveyed. The reference channel receives the direct signal from the transmitter, which is used to cancel the multipath at the surveillance channel and evaluate the delayDoppler cross-correlation function (2D-CCF) between the surveillance and the reference signal.

$$
|\chi(\tau, v)|^{2}=\left|\int_{-\infty}^{+\infty} x(t) \dot{s}^{\prime}(t-\tau) \exp (j 2 \pi v t) \mathrm{d} t\right|^{2},
$$

where $x(t)$ is the target echo signal, $s(t)$ is the reference signal, and $v$ and $\tau$ are the Doppler frequency shift and the sample time-delay parameters, respectively.

In practice, the surveillance channel collects a mixture of Doppler-shifted and time-delayed replicas of the reference signal from the transmitter of opportunity, involving target echoes, direct signal, strong clutter, or multipath echoes, and the CCI signals from other nuisance illuminators. Different approaches have been proposed to cope with direct signal and multipath $[12,24,26,32]$. However, with respect to the cancellation of direct signal and multipath, CCI rejection requires different techniques for preprocessing before matched filtering. Otherwise, detecting targets' performance and capabilities, incredibly weak targets, will degrade a lot in a PBR. The PLCA method for signal preprocessing to remove the CCI in a PBR will be discussed in what follows.

\section{The Probabilistic Latent Component Analysis Method}

3.1. Probabilistic Latent Component Analysis. Probabilistic latent component analysis (PLCA) is a straightforward extension of probabilistic latent semantic indexing (PLSI) [33] which deals with an arbitrary number of dimensions. The basic model is defined as follows [30]:

$$
P(x)=\sum_{z} P(z) \prod_{j=1}^{N} P\left(x^{j} \mid z\right),
$$

where $P(x)$ denotes a distribution over the $N$-dimensional random variable $x$ and $x^{j}$ is $j^{\prime}$ th dimension. $z$ is a latent variable, and $P\left(x^{j} \mid z\right)$ is one-dimensional distribution. Effectively this model represents a mixture of marginal distribution products to approximate an $N$ dimensional distribution. Subsequent processing is to discover the most appropriate marginal distributions. To perform the estimation of the marginals $P\left(x^{j} \mid z\right)$, the EM algorithm is considered. 


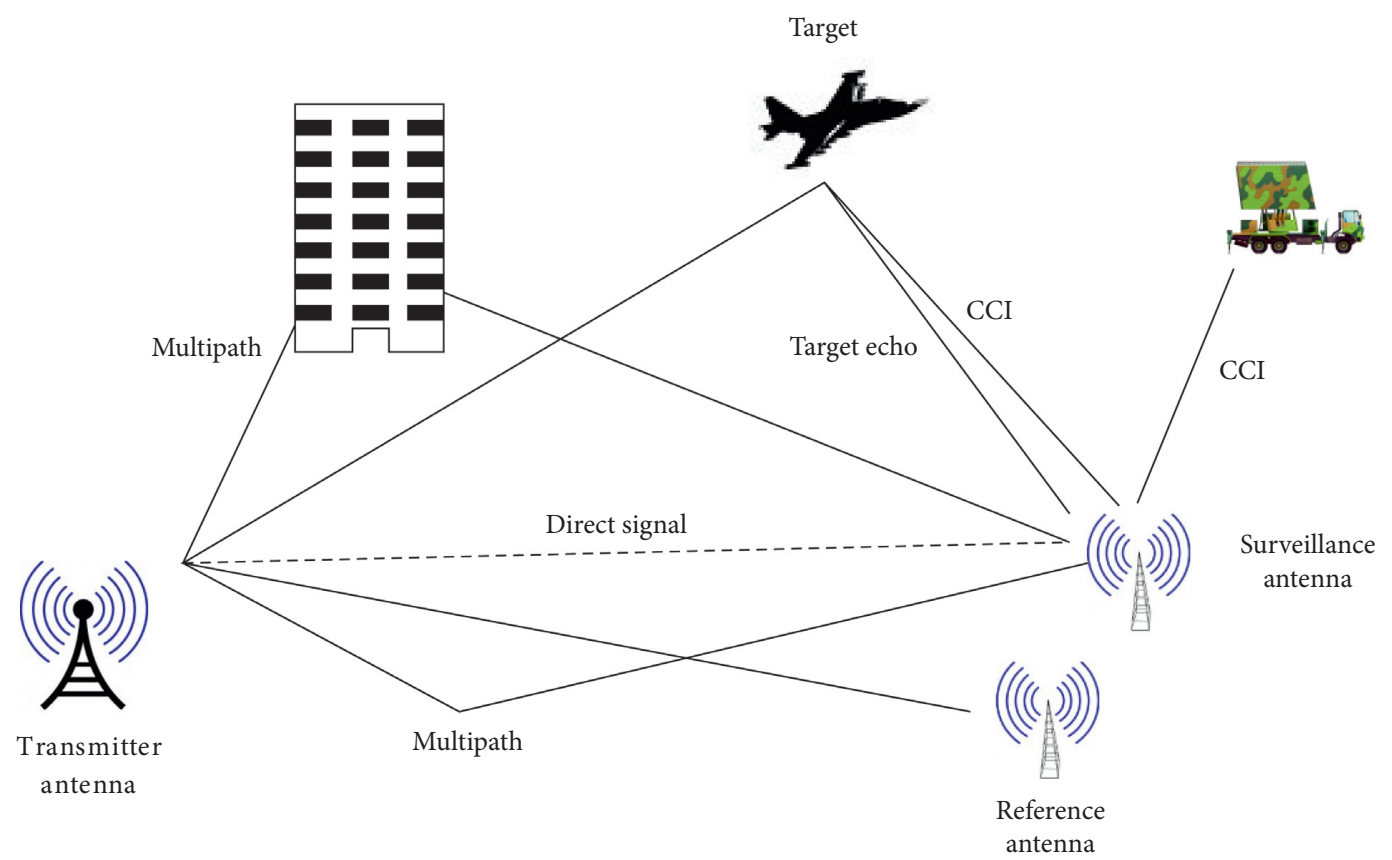

FIgURE 1: PBR geometry.

(i) E step: as seen from (2), $P\left(x^{j} \mid z\right)$ is independent of each other:

$$
P(x \mid z)=\prod_{j=1}^{N} P\left(x^{j} \mid z\right) .
$$

In this step, the posterior probability of the latent variable $z$ is estimated as follows:

$$
P(z \mid x)=\frac{P(z) \prod_{j=1}^{N} P\left(x^{j} \mid z\right)}{\sum_{z^{\prime}} P\left(z^{\prime}\right) \prod_{j=1}^{N} P\left(x^{j} \mid z^{\prime}\right)} .
$$

(ii) M step: a new $z$ distribution is obtained as follows:

$$
P(z)=\int P(x) P(z \mid x) \mathrm{d} x
$$

Define

$$
P^{*}\left(x^{j} \mid z\right)=\int \cdots \int P(x) P(z \mid x) \mathrm{d} x^{k}, \quad \forall k \neq j .
$$

A new and more accurate estimation of $z$ is obtained as follows:

$$
P\left(x^{j} \mid z\right)=\frac{P^{*}\left(x^{j} \mid z\right)}{P(z)} .
$$

A converging solution for the marginals $P\left(x^{j} \mid z\right)$ along each dimension $j$ and the latent variable priors $P(z)$ will be obtained by repeating the above steps in an alternating manner multiple times. It should be noted that $P(x)$ and $z$ are both discrete in practical application.
3.2. The Probabilistic Latent Component Analysis Method. Actually, we can consider the removal of CCI as reconstruction and extraction of Doppler-shifted and timedelayed replicas of the reference signal from the mixture received in the surveillance channel. Moreover, the reference channel offers us a source of training data for PLCA without increasing the complexity of the receiving system. As a consequence, PLCA is modified and employed for the removal of CCI. The PLCA method for the removal of CCI explicitly models the collected signal as a mixture of marginal distribution products. The estimation of the most appropriate marginal distributions, performed using the expectation-maximization algorithm, is employed selectively to reconstruct and extract the desired signal. Eventually, the disturbance suppression is achieved.

To begin with, perform the short-time Fourier transform of a signal $s(t)$ :

$$
\operatorname{STFT}_{s}(t, f)=\int_{-\infty}^{\infty}\left[s(\tau) \gamma^{*}(\tau-t)\right] e^{-j 2 \pi f \tau} \mathrm{d} \tau .
$$

The spectrogram of $s(t)$ can be treated as the distribution of signal energy over time and frequency domain, $\widehat{P}(f, t)=\operatorname{abs}\left[\operatorname{STFT}_{s}(t, f)\right]$.

Proper normalization will make the spectrogram of signal a distribution of energy across the time-frequency plane $P(f, t)$. Adopting this view allows us to employ PLCA directly for analysis of the signal. The $2 \mathrm{D}$ model of PLCA is defined as follows:

$$
P(f, t)=\sum_{z} P(z) P(f \mid z) P(t \mid z)
$$

where $P(f, t)$ is a magnitude spectrogram of signal and $P(f \mid z)$ and $P(t \mid z)$ are frequency marginals and time 

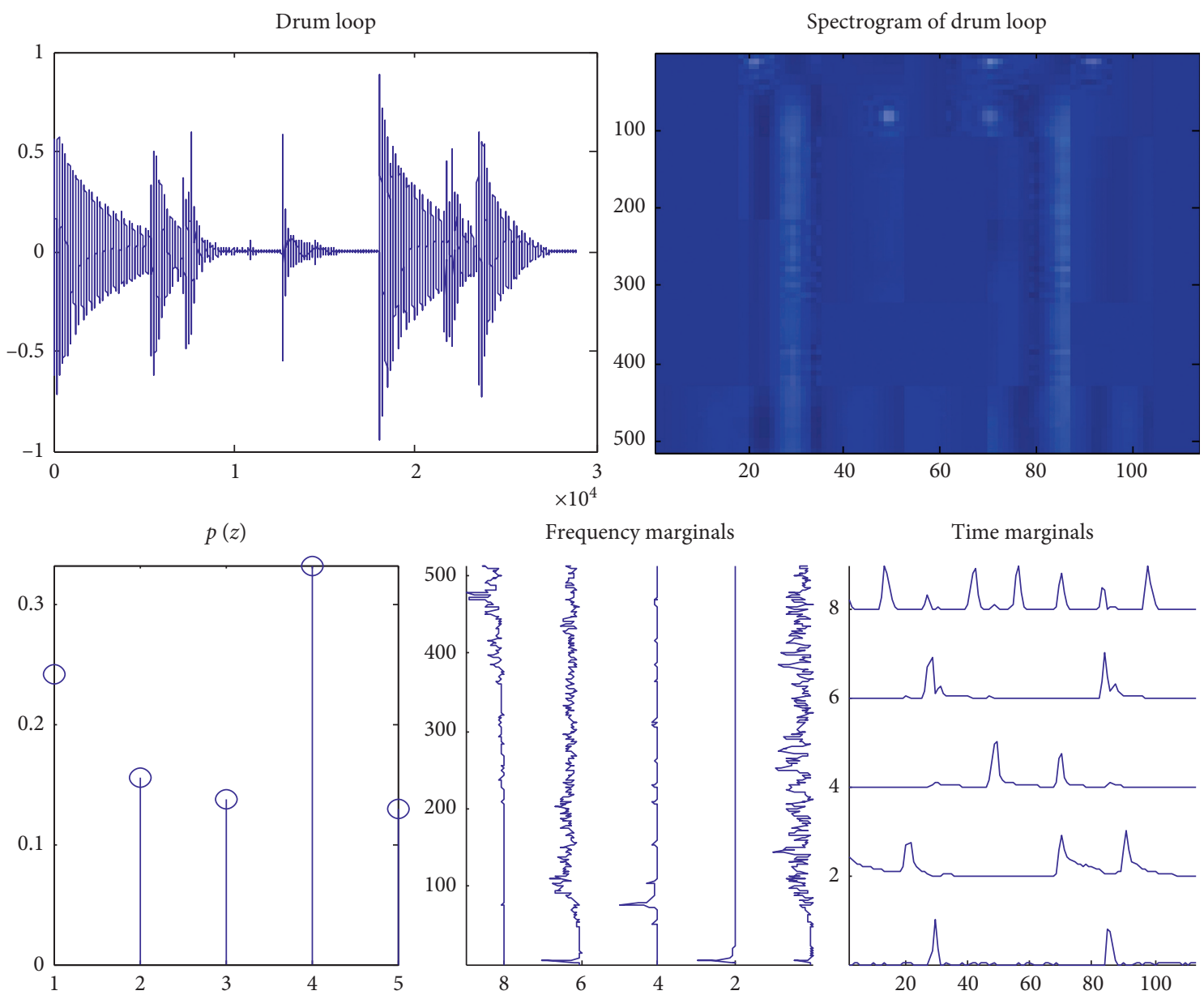

FIgUre 2: Application of PLCA on the spectrogram of a drum loop.

marginals, respectively. The marginals will contain a dictionary of spectra which can best describe the signal represented by the input spectrogram. The application of PLCA on the spectrogram of a drum loop and its corresponding marginals are shown in Figure 2. Reconstruction of different frequency marginals and time marginals will lead to extraction of different components of the drum loop.

Notice that these marginals can be used as a model of a class of acoustic signals. Likewise, we can model the signal received by the surveillance channel as these marginals. Next, we will apply the PLCA to the CCI removal. The total collected signal in the surveillance channel can be given by

$$
\begin{aligned}
x(t) & =A_{\text {surv }} d(t)+\sum_{m=1}^{M} a_{m} d\left(t-\tau_{m}\right) e^{j 2 \pi f_{\mathrm{d} m} t} \\
& +\sum_{i=1}^{N} c_{i} d\left(t-\tau_{\mathrm{ci}}\right)+\sum_{k=1}^{K} j_{k}(t)+n_{\text {surv }}(t),
\end{aligned}
$$

where $d(t)$ is the complex envelope of the direct signal (a delayed replica of the transmitted signal from the illuminator of opportunity); $A_{\text {surv }}$ is the complex amplitude of the direct signal received via the side/back lobe of the surveillance; $a_{m}, \tau_{m}$, and $f_{0 m}$ are the complex amplitude, the delay (with respect to the direct signal), and the Doppler frequency of the $m$ th target $(m=1, \ldots, M) ; c_{i}$ and $\tau_{c i}$ are the complex amplitude and the delay (with respect to the direct signal) of the $i$ th stationary ground scatter $(i=1, \ldots, N) ; j_{k}(t)$ is the $k$ th CCI contribution from other nuisance illuminators $(j=1, \ldots, K)$; and $n_{\text {surv }}(t)$ is the thermal noise contribution at the surveillance antenna.

Further, we define

$$
\begin{aligned}
S(t)= & A_{\text {surv }} d(t)+\sum_{m=1}^{M} a_{m} d\left(t-\tau_{m}\right) e^{j 2 \pi f_{\mathrm{d} m} t} \\
& +\sum_{i=1}^{N} c_{i} d\left(t-\tau_{\mathrm{ci}}\right)+n_{\mathrm{surv}}(t), \\
J(t) & =\sum_{k=1}^{K} j_{k}(t) .
\end{aligned}
$$

After substitution of (11) and (12) into (10), we have

$$
x(t)==S(t)+J(t) .
$$

The complex envelope of the signal at the reference channel is 


$$
s_{\text {ref }}(t)=A_{\text {ref }} d(t)+n_{\text {ref }}(t),
$$

where $A_{\text {ref }}$ is the complex amplitude and $n_{\text {ref }}(t)$ is the thermal noise contribution at the reference antenna. Since the direct signal is received by the main lobe of the reference antenna, for simplification of analysis it is assumed that the reference signal is free of multipath. It is also noted that the effect of multipath on the reference channel has been analyzed in [34] where a potential algorithm for its removal has been introduced.

The distribution $P_{x}(f, t)$ of signal energy across timefrequency plane will be obtained by performing the shorttime Fourier transform of $x(t)$. The relevance between $s_{\text {ref }}(t)$ and $S(t)$ implies that the data from the reference channel can be utilized as training data. The posterior probability of the latent variable $z$ is estimated as follows:

$$
P_{s}(z \mid f, t)=\frac{P_{s}(z) P_{s}(f \mid z) P_{s}(t \mid z)}{\sum_{z^{\prime}} P\left(z^{\prime}\right) P_{s}\left(f \mid z^{\prime}\right) P_{s}\left(t \mid z^{\prime}\right)} .
$$

We can estimate the frequency marginals $P_{s}(f \mid z)$ to reconstruct $S(t)$ by performing the EM algorithm mentioned above. According to (11), (12), and (13), the collected signal in the surveillance channel can be treated as a mixture of Doppler-shifted and time-delayed replicas of the reference signal from the transmitter of opportunity and the CCI signals from other nuisance illuminating sources. Therefore, the removal of CCI can be considered as the equivalent of reconstruction and extraction of Doppler-shifted and timedelayed replicas of the reference signal from the mixture. Thus, our objective is to discover a dictionary of $P_{J}(f \mid z)$ and $P_{J}(t \mid z)$ to describe the source of CCI. Simultaneously, the solution of the time marginals $P_{s}(t \mid z)$ will be used to reconstruct $S(t)$.

To solve $P_{J}(f \mid z), P_{J}(t \mid z)$, and $P_{s}(t \mid z)$, extend the frequency marginals of the training data

$$
P_{x}(f \mid z)=\left[\begin{array}{ll}
P_{S}(f \mid z) & P_{J}(f \mid z)
\end{array}\right] .
$$

For a fixed $z$,

$$
P(z \mid f, t)=\frac{P(z) P_{x}(f \mid z) P_{x}(t \mid z)}{\sum_{z^{\prime}} P\left(z^{\prime}\right) P_{x}\left(f \mid z^{\prime}\right) P_{x}\left(t \mid z^{\prime}\right)} .
$$

The new latent variable prior is obtained as follows:

$$
P(z)=\iint P_{x}(f, t) P(z \mid f, t) \mathrm{d} f \mathrm{~d} t .
$$

Define

$$
\begin{aligned}
P_{x}^{*}(f \mid z) & =\int P_{x}(f, t) P(z \mid f, t) \mathrm{d} t, \\
P_{x}^{*}(t \mid z) & =\int P_{x}(f, t) P(z \mid f, t) \mathrm{d} f .
\end{aligned}
$$

Compute the new marginals

$$
\begin{aligned}
& P_{x}(f \mid z)=\frac{P_{x}^{*}(f \mid z)}{P(z)}=\left[\begin{array}{ll}
P_{s}(f \mid z) & \left.P_{J}(f \mid z)\right]
\end{array},\right. \\
& P_{x}(t \mid z)=\frac{P_{x}^{*}(t \mid z)}{P(z)}=\left[\begin{array}{l}
P_{s}(t \mid z) \\
P_{J}(t \mid z)
\end{array}\right] .
\end{aligned}
$$

Repeating (17), (18), (20), and (21) in an alternating manner multiple times yields a converging solution for $P_{J}(f \mid z), P_{J}(t \mid z)$, and $P_{s}(t \mid z)$. Reconstruction of the signal is obtained as follows:

$$
P_{s}(f, t)=\sum_{z} P_{s}(f \mid z) P(z) P_{s}(t \mid z) .
$$

As a consequence, the CCI from other nuisance illuminators will be removed.

\section{Simulation Results}

In this section, the developed PLCA method's performance is verified using DVB-T signals in $2 \mathrm{k}$ mode for simulations. The numerical values for the OFDM parameters are listed in Table 1. The number of OFDM symbols is 30. Figures 3(a)3 (d) show the spectrum, 2D autoambiguity function, and the 2D autoambiguity function's major cuts at zero range and zero Doppler. The DVB-T-modulated signal achieves a fair range resolution due to its high bandwidth. Besides, some side peaks in the $2 \mathrm{D}$ autoambiguity function of a DVB-T-modulated signal can be suppressed by the developed algorithm in [35], thus yielding a reduction in false alarms.

For emphasis on evaluating the performance and capabilities of CCI removal of the PLCA method, we simplify the scenario of simulation where two targets with the identical signal-to-noise ratio (SNR) of $5 \mathrm{~dB}$ are placed at $(15 \mathrm{~km}, 600 \mathrm{~Hz})$ and $(18 \mathrm{~km},-300 \mathrm{~Hz})$, respectively, in the range-Doppler domain in the absence of direct signal and multipath clutter which can be cancelled by the examined approach in [32] in the subsequent processing.

Assume that there are two nuisance sources, transmitting noise-amplitude-modulated signal and noise-frequency-modulated signal, respectively, and both the ratios of powers of target echo signal and CCI (SIR) are $-30 \mathrm{~dB}$. If we do not take measures to remove the disturbance in the first step using some method, the subsequent target detection will be affected severely. The comparison of Figures 4 and 5 confirms this point. The two target echoes are entirely submerged in the background of CCI from other nuisance illuminators without removal.

Now, the PLCA method is utilized for the removal of CCI from other nuisance illuminators. The reference signal is exploited as training data modeled using 20 marginals while the collected signal in the surveillance channel was modeled using 40 marginals. The number of iterations for the EM algorithm is 200. Figure 6 shows the target echos, the corrupted signal in the surveillance channel, and the 
TABLE 1: Numerical values for the OFDM parameters for the $2 \mathrm{k}$ mode.

\begin{tabular}{lc}
\hline Parameter & $2 \mathrm{k}$ mode \\
\hline Sampling frequency & $64 / 7 \mathrm{MHz}$ \\
Number of carriers & 1705 \\
Bandwidth & $7.61 \mathrm{MHz}$ \\
Duration of symbol part & $224 \mu \mathrm{s}$ \\
Duration of guard interval & $14 \mu \mathrm{s}$ \\
\hline
\end{tabular}

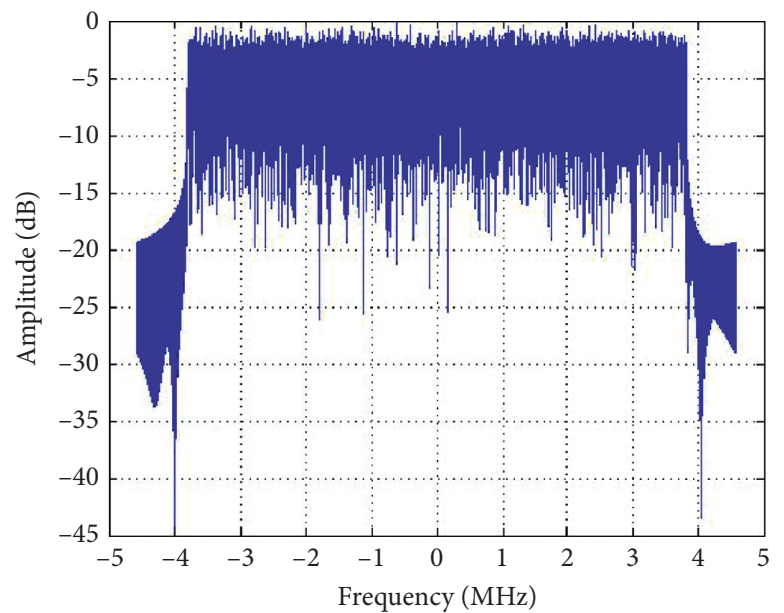

(a)

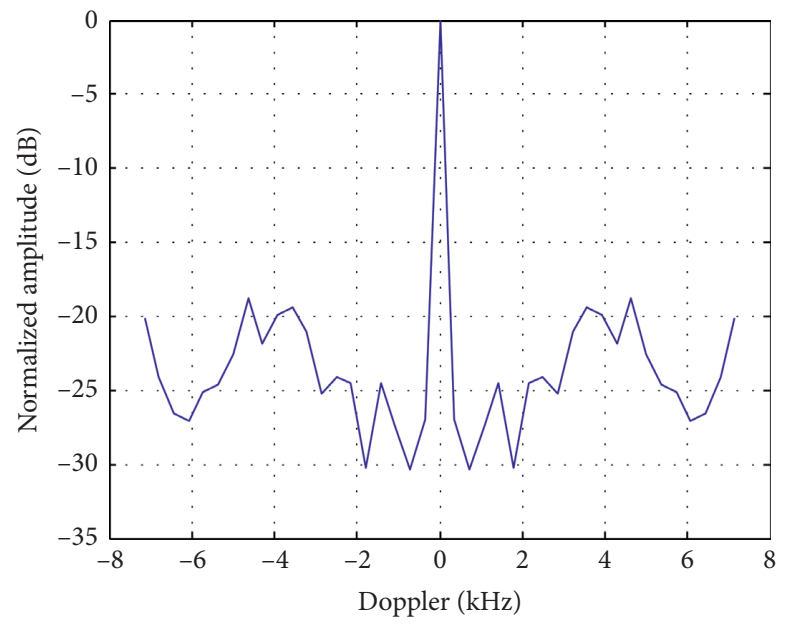

(c)

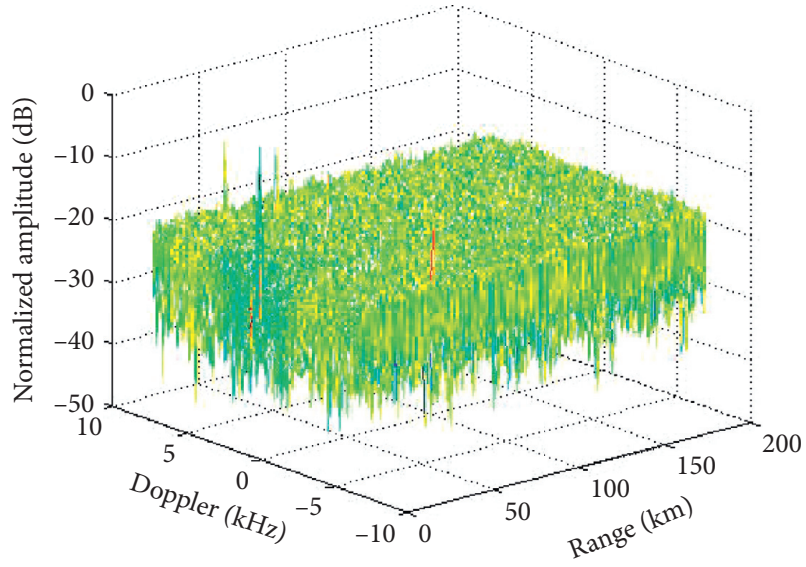

(b)

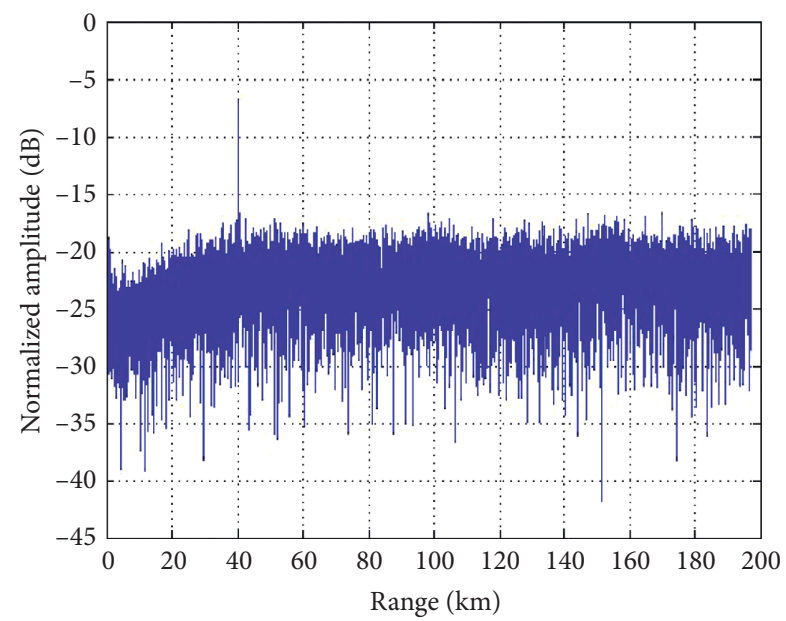

(d)

Figure 3: Spectrum and ambiguity function of DVB-T signal. (a) Spectrum; (b) 2D autoambiguity function; (c) zero range cut of autoambiguity function; (d) zero Doppler cut of autoambiguity function.

reconstruction and extraction of the desired signal by using the PLCA method, respectively, from top to bottom when $\mathrm{SIR}=-30 \mathrm{~dB}, \mathrm{SNR}=5 \mathrm{~dB}$, and $\mathrm{SNRre}=5 \mathrm{~dB}$ (the ratio of powers of the reference signal and noise). The 2D-CCF between the reconstructed signal by using the PLCA method and the reference signal indicates that the CCI from other 


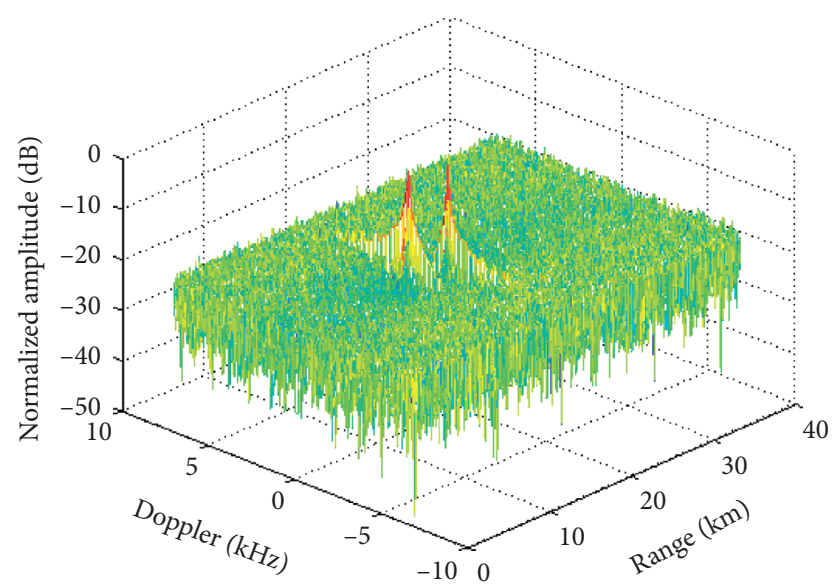

FIGURE 4: 2D-CCF in the absence of CCI from other nuisance illuminators.

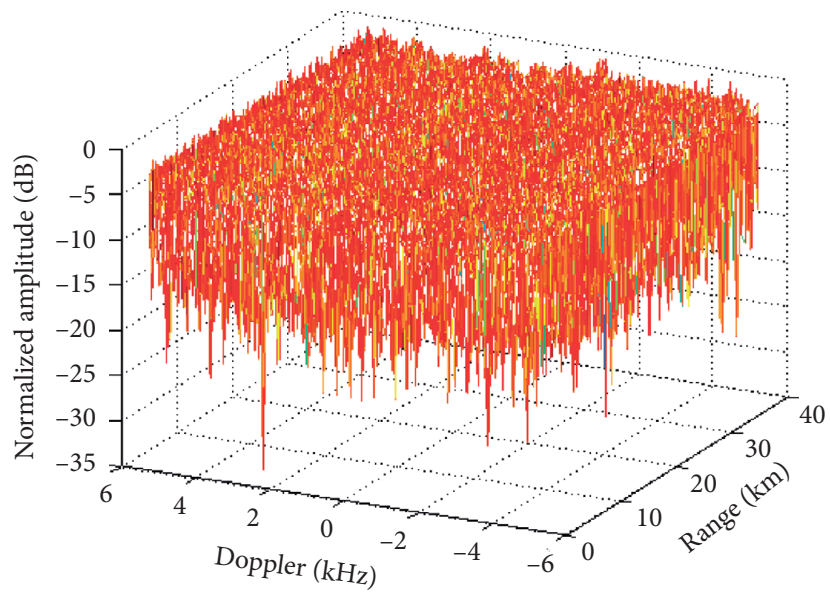

FIgURE 5: 2D-CCF in the presence of CCI from other nuisance illuminators.

nuisance illuminators is removed effectively, and the target echoes stand out in Figure 7.

For further evaluation of the proposed PLCA method's performance in comparison with traditional methods, we employ the methods based on LMS adaptive filter and wavelet transform for CCI removal. Wavelet decomposition is performed at level 2 with a selection of heursure threshold and the orthogonal wavelet sym8 is adopted. We specify the order and step size of the LMS filter as 5 and 0.001 , respectively. Besides, we define the signal-to-distortion ratio (SDR) as follows:

$$
\mathrm{SDR}=10 \log _{10} \frac{\|s(t)\|_{2}^{2}}{\left\|s(t)-s^{\prime}(t)\right\|_{2}^{2}},
$$

where $s(t)$ is the desired signal and $s^{\prime}(t)$ is the reconstruction of $s(t)$. The comparison of CCI removal performance of the PLCA method regarding traditional methods, including LMS adaptive filter and wavelet transform, is depicted schematically in Figure 8. Obviously, the PLCA method is superior to the LMS method and the wavelet method. Moreover, the traditional methods can barely work to remove CCI and yield 

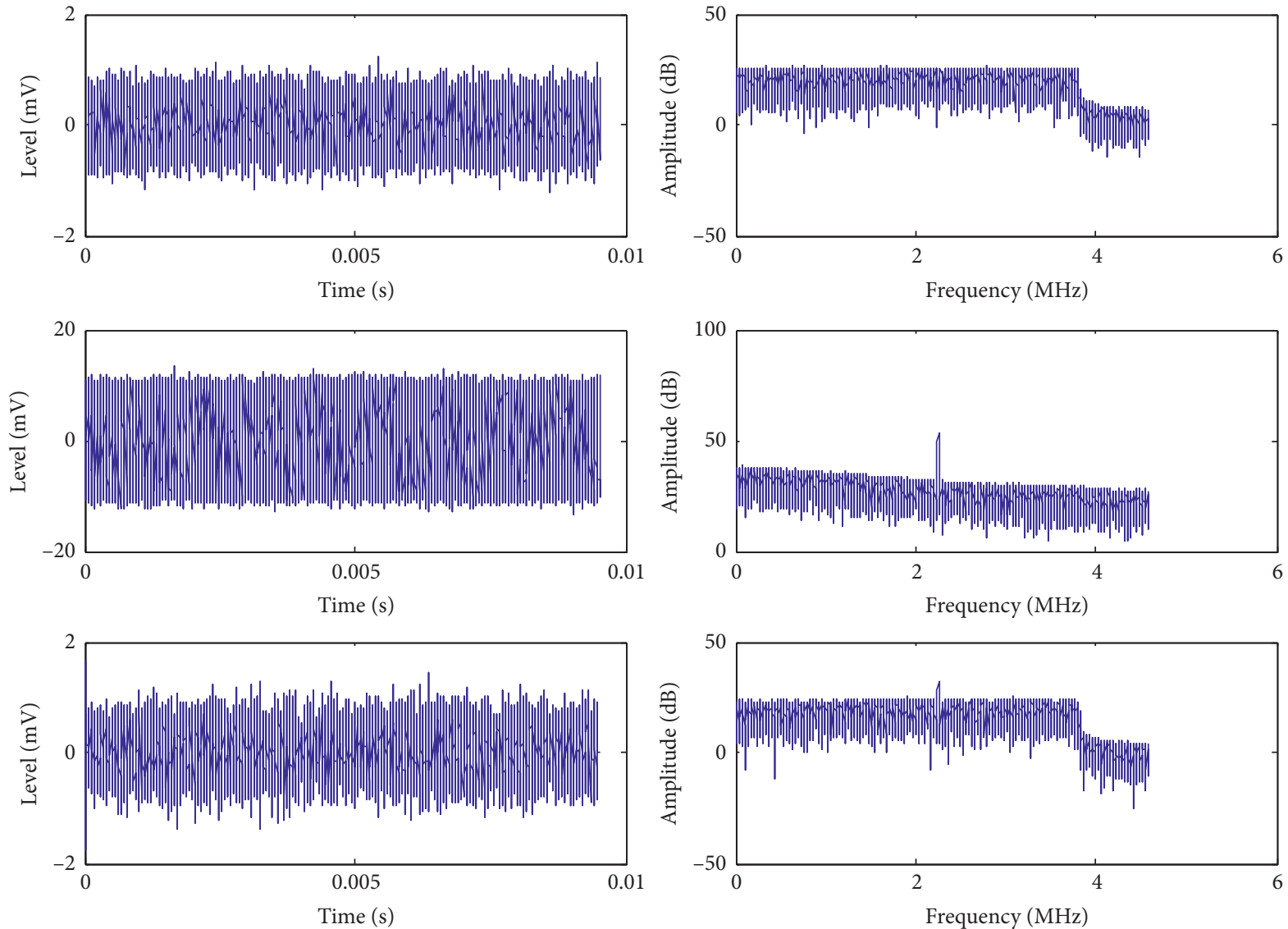

FIGURE 6: Target echos, corrupted signal, and reconstruction of the desired signal, respectively, from top to bottom with SIR $=-30 \mathrm{~dB}$, $\mathrm{SNR}=5 \mathrm{~dB}$, and SNRre $=5 \mathrm{~dB}$

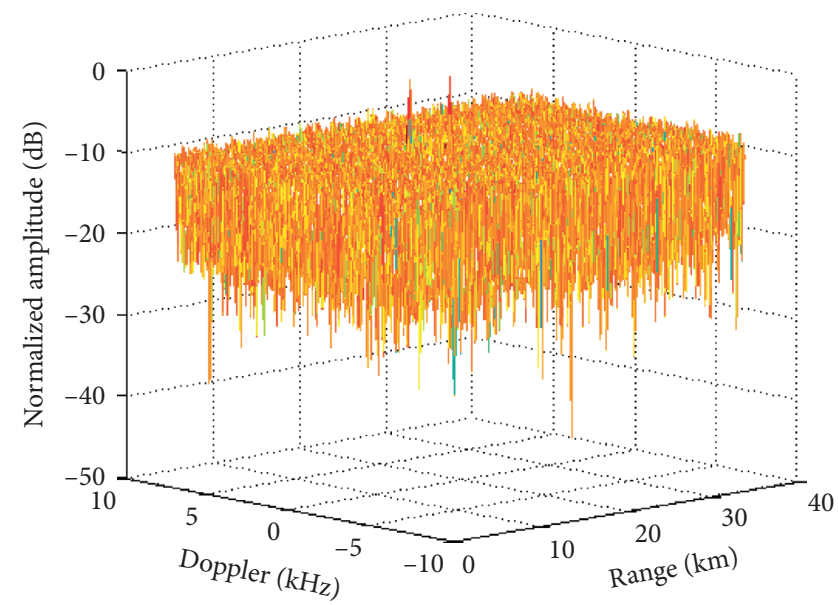

Figure 7: 2D-CCF with disturbance removal using the PLCA method.

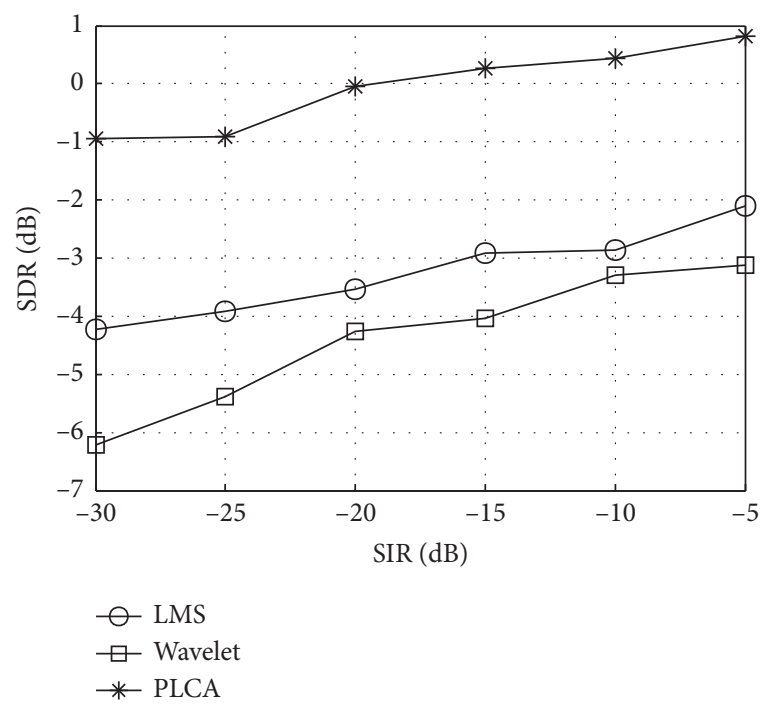

FIGURE 8: Comparison of SDR versus SIR with different methods. 


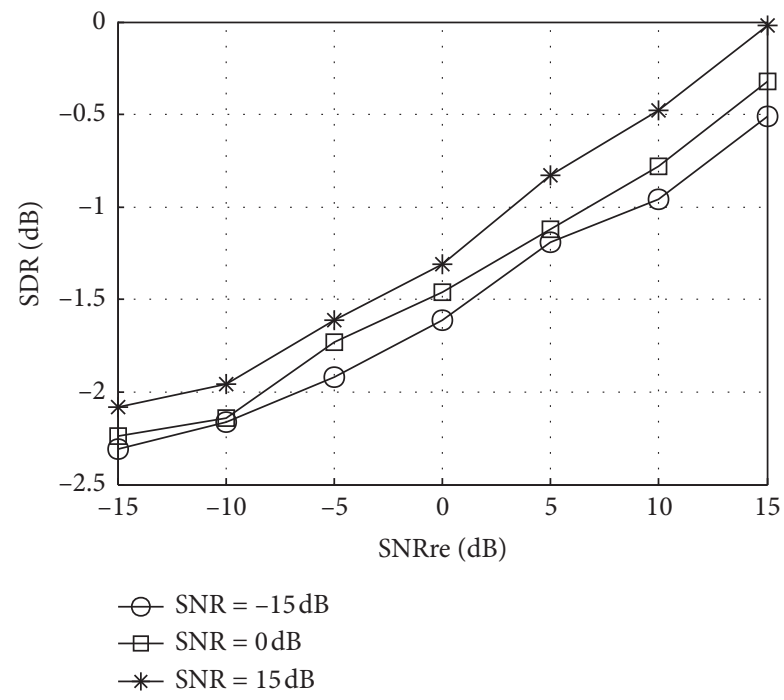

FIGURE 9: SDR versus SNRre with different SNRs when $\mathrm{SIR}=-25 \mathrm{~dB}$.

contribution to target detection, significantly when CCI exceeds target echoes over $20 \mathrm{~dB}$ in power. Figure 9 shows that a wide variety in SNR does not bring a significant change in SDR, while SDR changes sensitively with SNRre for the PLCA method. That is because the reference signal is exploited as training data for modeling and reconstruction of the desired signal in the corrupted signal in the surveillance channel. The purity of the reference signal is significant for the effectiveness of the PLCA method.

\section{Conclusions}

In this paper, the problem of removal of CCI from other nuisance illuminators was investigated in a PBR. The PLCA method was proposed and treated the removal of CCI as the reconstruction and extraction of Doppler-shifted and timedelayed replicas of the reference signal. Finally, some methods were compared by simulation, and it was demonstrated that the proposed PLCA method was effective and superior to the other methods.

\section{Data Availability}

The data used to support the findings of this study are available from the corresponding author upon request.

\section{Conflicts of Interest}

The authors declare no conflicts of interest.

\section{Authors' Contributions}

T. Y. proposed the method, performed the experiments, and wrote the paper; X. W. designed the experiments and analyzed the data; T. Y. and W. T. made the discussion; C. Z. and $\mathrm{W}$. T. gave the instructional suggestions.

\section{Acknowledgments}

This work was supported by the National Nature Science Foundation of China (no. 61803379) and the General
Program Supporting Fund of China Postdoctoral Science Foundation (no. 2017M613370).

\section{References}

[1] D. K. P. Tan, H. Sun, Y. Lu, and M. Lesturgie, "Space-time interference analysis and suppression for airborne passive radar using transmissions of opportunity," IET Radar, Sonar \& Navigation, vol. 8, no. 2, pp. 142-152, 2014.

[2] X. Zhang, H. Li, and B. Himed, "Maximum likelihood delay and doppler estimation for passive sensing," IEEE Sensors Journal, vol. 19, no. 1, pp. 180-188, 2018.

[3] T. Shan, S. Liu, R. Tao, and G. Zhang, "Experiment demonstration of micro-doppler detection of rotor blades with passive coherent location based on digital video broadcast," Journal of Communications Technology and Electronics, vol. 59, no. 11, pp. 1215-1224, 2014.

[4] A. Zaimbashi, "Target detection in analog terrestrial TV-based passive radar sensor: joint delay-doppler estimation," IEEE Sensors Journal, vol. 17, no. 17, pp. 5569-5580, 2017.

[5] R. Tharmarasa, M. Subramaniam, N. Nadarajah, T. Kirubarajan, and M. McDonald, "Multitarget passive coherent location with transmitter-origin and target-altitude uncertainties," IEEE Transactions on Aerospace and Electronic Systems, vol. 48, no. 3, pp. 2530-2550, 2012.

[6] S. Gogineni and A. Nehorai, "Target estimation using sparse modeling for distributed MIMO radar," IEEE Transactions on Signal Processing, vol. 59, no. 11, pp. 5315-5325, 2011.

[7] M. Malanowski, M. S. Greco, A. Bernard, R. Haugen, R. Plšek, and D. W. O'Hagan, "Land and sea clutter from FM-based passive bistatic radars," IET Radar, Sonar \& Navigation, vol. 8, no. 2, pp. 160-166, 2014.

[8] C. Liu and W. Chen, "Sparse self-calibration imaging via iterative MAP in FM-based distributed passive radar," IEEE Geoscience and Remote Sensing Letters, vol. 10, no. 3, pp. 538-542, 2013.

[9] D. Poullin, "Passive detection using digital broadcasters (DAB, DVB) with COFDM modulation," IEE Proceedings-Radar, Sonar and Navigation, vol. 152, no. 3, pp. 143-152, 2005.

[10] T. Shan, Y. Feng, R. Tao, S. Liu, M. G. Amin, and Y. D. Zhang, "Efficient architecture and hardware implementation of 
coherent integration processor for digital video broadcastbased passive bistatic radar," IET Radar, Sonar \& Navigation, vol. 10, no. 1, pp. 97-106, 2016.

[11] J. E. Zhang, H. A. Harms, S. J. Searle, and L. Davis, "DVB-T passive radar signal processing," IEEE Transactions on Signal Processing, vol. 61, no. 8, pp. 2116-2126, 2013.

[12] J. Raout, A. Santori, and E. Moreau, "Space-time clutter rejection and target passive detection using the APES method," IET Signal Processing, vol. 4, no. 3, pp. 298-304, 2010.

[13] M. Radmard, S. M. Karbasi, and M. M. Nayebi, "Data fusion in MIMO DVB-T-based passive coherent location," IEEE Transactions on Aerospace and Electronic Systems, vol. 49, no. 3, pp. 1725-1737, 2013.

[14] L.-P. Gill, D. Grenier, and J.-Y. Chouinard, "Use of XMTM radio satellite signal as a source of opportunity for passive coherent location," IET Radar, Sonar \& Navigation, vol. 5, no. 5, pp. 536-544, 2011.

[15] P. Stinco, M. S. Greco, F. Gini, and M. Rangaswamy, "Ambiguity function and Cramér-Rao bounds for universal mobile telecommunications system-based passive coherent location systems," IET Radar, Sonar \& Navigation, vol. 6, no. 7, pp. 668-678, 2012.

[16] S. Gogineni, M. Rangaswamy, B. D. Rigling, and A. Nehorai, "Cramér-rao bounds for UMTS-based passive multistatic radar," IEEE Transactions on Signal Processing, vol. 62, no. 1, pp. 95-106, 2014.

[17] A. D. Maio, G. Foglia, N. Pasquino, and M. Vadursi, "Measurement and comparative analysis of clutter for GSM and UMTS passive radars," IET Radar Sonar and Navigation, vol. 4, no. 3, pp. 412-423, 2010.

[18] W.-Q. Wang, "GPS-based time \& phase synchronization processing for distributed SAR," IEEE Transactions on Aerospace and Electronic Systems, vol. 45, no. 3, pp. 10401051, 2009.

[19] F. Colone, K. Woodbridge, H. Guo, D. Mason, and C. J. Baker, "Ambiguity function analysis of wireless LAN transmissions for passive radar," IEEE Transactions on Aerospace and Electronic Systems, vol. 47, no. 1, pp. 240-264, 2011.

[20] F. Colone, P. Falcone, C. Bongioanni, and P. Lombardo, "WiFi-based passive bistatic radar: data processing schemes and experimental results," IEEE Transactions on Aerospace and Electronic Systems, vol. 48, no. 2, pp. 1061-1079, 2012.

[21] K. Chetty, G. E. Smith, and K. Woodbridge, "Through-the-wall sensing of personnel using passive bistatic WiFi radar at standoff distances," IEEE Transactions on Geoscience and Remote Sensing, vol. 50, no. 4, pp. 1218-1226, 2012.

[22] Q. Wang, C. Hou, and Y. Lu, "An experimental study of WiMAX-based passive radar," IEEE Transactions on Microwave Theory and Techniques, vol. 58, no. 12, pp. 3502-3510, 2010.

[23] Z. Zhao, X. Wan, D. Zhang, and F. Cheng, "An experimental study of HF passive bistatic radar via hybrid sky-surface wave mode," IEEE Transactions on Antennas and Propagation, vol. 61, no. 1, pp. 415-424, 2013.

[24] K. S. Kulpa and Z. Czekała, "Masking effect and its removal in PCL radar," IEE Proceedings-Radar, Sonar and Navigation, vol. 152, no. 3, pp. 174-178, 2005.

[25] S. Liu, Y. Ma, and Y. Huang, "Sea clutter cancellation for passive radar sensor exploiting multi-channel adaptive filters," IEEE Sensors Journal, vol. 19, no. 3, pp. 982-995, 2019.

[26] F. Colone, D. W. O’Hagan, P. Lombardo, and C. J. Baker, “A multistage processing algorithm for disturbance removal and target detection in passive bistatic radar," IEEE Transactions on Aerospace and Electronic Systems, vol. 45, no. 2, pp. 698$722,2009$.

[27] X. Gao, X. Wang, X. Li, and D. Tao, "Transfer latent variable model based on divergence analysis," Pattern Recognition, vol. 44, no. 10-11, pp. 2358-2366, 2011.

[28] J. Gao, J. Zhang, and D. Tien, "Relevance units latent variable model and nonlinear dimensionality reduction," IEEE Trans on Neural Networks, vol. 21, no. 1, pp. 123-135, 2010.

[29] X. Gao, X. Wang, D. Tao, and L. Xuelong, "Supervised Gaussian process latent variable model for dimensionality reduction," IEEE Trans on Systems, Man, and Cybernetics, Part B: Cybernetics.vol. 41, no. 2, pp. 425-434, 2011.

[30] P. Smaragdis, B. Raj, and M. Shashanka, "Supervised and semi-supervised separation of sounds from single-channel mixtures," in Proceedings of the ICA Conference on Independent Component Analysis and Signal Separation, pp. 414-421, London, UK, September 2007.

[31] T. Ying, G. Huang, and C. Zhou, "Probabilistic latent component analysis for radar signal detection," in Proceedings of the 2013 6th Conference on Image and Signal Processing, Hangzhou, China, September 2013.

[32] T. Ying, G. Huang, W. Zuo, H. Shan, and J. Gao, "Weak target detection method based on hypothesis test theory in noncooperative passive detection," Acta Aeronautica et Astronautica Sinica, vol. 37, pp. 626-636, 2016.

[33] T. Hofmann, "Probabilistic latent semantic indexing," in Proceedings of the 22nd Annual International ACM SIGIR Conference on Research and Development in Information Retrieval, pp. 50-57, Berkeley, CA, USA, 1999.

[34] F. Colone, R. Cardinali, P. Lombardo et al., "Space-time constant modulus algorithm for multipath removal on the reference signal exploited by passive bistatic radar," IET Radar, Sonar \& Navigation, vol. 3, no. 3, pp. 253-264, 2009.

[35] F. Colone, D. Langellotti, and P. Lombardo, "DVB-T signal ambiguity function control for passive radars," IEEE Transactions on Aerospace and Electronic Systems, vol. 50, no. 1, pp. 329-347, 2014. 